\title{
Anemia del lactante desnutrido marásmico
}

\author{
Manuel Olivares $\mathrm{G}^{\mathbf{1}}$; Eva Hertrampf D. ${ }^{1}$; \\ Patricia Chadud $\mathbf{M}^{2}$
}

\begin{abstract}
Anemia in marasmic infants
To determine the prevalence and etiology of anemia in infants with marasmic malnutrition, 66 malnourished infants (6 to 21 months of age) admitted to a nursing home for nutritional recovery, whose weight for age was under -2 standard deviations of the NCHS tables were studied. The cause of marasmus was decreased food intake. Those infants with albumin $<3.5 \mathrm{~g} / \mathrm{d}$ l or serum protein $<6 \mathrm{~g} / \mathrm{dl}$ had been previously excluded. Whithin 3 days of admission a fasting venipuncture was performed to determine hemoglobin ( $\mathrm{Hb}$ ), serum iron (Fe), total iron binding capacity, transferrin saturation (Sat\}, free erythrocyte protoporphyrin (FEP), sorum ferritin (SF), serum folate (SFI) and erythrocyte folate (EF). Fifty three percent of the subjects presented a mild anemia. The prevalence of abnormal values was $64.6 \%$ for Sat $\langle 10 \%, 20.4 \%$ for FEP $>120 \mu \mathrm{g} / \mathrm{d}$ l RBC $16-11$ months of age 1 and $>100$ $\mu \mathrm{g} / \mathrm{dl}$ RBC (over 11 months of age), $19 \%$ for SF $<10 \mu \mathrm{g} / \mathrm{l}, 10.3 \%$ for SFI $<3 \mu \mathrm{g} / \mathrm{l}$ and $21.1 \%$ tor EF $<140 \mu \mathrm{g} / \mathrm{l}$ RBC. Hemoglobin concentration was correlated with weight/age $Z$ score $(r=0.35 ; p<0.005)$, height/age $Z$ score $i r=0.26: p<0.04\}$, Fe $\{r=0.24: p=0.05)$ and FEP $(r=-0.55: p<0.0001)$. in $46.7 \%$ of the anemic subjects the reduction in hemoglobin concentration is exclusively explained by the calorie and protein restriction. Other contributory mechanisms were iron deficiency, folic acid deficiency and both iron and folate deficiencies in $30 \%$, $16.7 \%$ and $6.7 \%$ of the anerric infants respectively.
\end{abstract}

(Key words: Malnutrition, iron deficiency, folate deficiency.)

La desnutrición proteica-calórica es frecuente en los países en vías de desarrollo. Esta condición se acompaña de anemia de etiologia multifactorial, variando las causas según el tipo de desnutrición de los paises ${ }^{1}$.

1. Unidad de Hematología, Instituto de Nutrición y Tecnología de los Alimentos, Universidad de Chie.

2. Tecnólogo Médico. Unidad de Hematología, Instituto de Nutrición y Tecnología de los Alimentos, Universidad de Chile.
El objetivo del presente trabajo fue describir la frecuencia de anemia y su coexistencia con algunos factores que podrían participar en su etiología, de lactantes desnutridos marásmicos, que han ingresado a los centros de recuperación nutricional de la Corporación para la Nutrición lnfantil (CONIN).

\section{Material y Métodos}

Previo consentimiento de sus padres se seleccionaron para el estudio 66 lactantes de 6 a 21 meses, ingre- 
sados a un centro de recuperación nutricional de CONIN de la ciudad de Santiago, cuyo peso para la edad fuese 2 desviaciones estándares bajo el promedio de las tablas del Centro Nacional de Estadísticas para Sahud de EUA, albúmina sérica superior a $3,5 \mathrm{~g} / \mathrm{dl}$ y proteína sérica mayor de $6 \mathrm{~g} / \mathrm{dt}$. Todos los sujetos tenían desnutrición primaria debida a ingesta insuficiente de alimentos. Dentro de los 3 primetos días desde la admisión, una vez estabilizado el sujeto y constatada la ausenciz de otras enfecmedades agregadas, se obtuvo en ayunas una muestra sanguínea venoss para medir: hemoglobina [Hb] (Coulter Counter, ZBI, Hialeah, Fl), hierro sérico [Fe], capacidad total de combinación de hierro [TIBC], saturación de la transferrina [Sat ] ${ }^{2}$, protoporfürina libre eritrocitaria [PLEl ${ }^{3}$, ferritina zérica [FS] (Gamma Dab 125I Radioimmunoassay, Travenol Laboratories, Cambridge, Mas.), folato sérico [FIS| y etitracitario [FIGR]".

Para los propósitos de este análisis se consideraron normales los siguientes valores: $H b>11 \mathrm{~g} / \mathrm{d}$, Sat $>$ $10 \%$, PLE $<120 \mathrm{~kg} / \mathrm{dl}$ eritrocitos entre 6 y 11 meses de edad y $\leqslant 100 \mathrm{~kg} / \mathrm{dl}$ eritrocitos desde los 12 meses, FS $>10 \mu \mathrm{g} / 1$, FlS $>3 \mu \mathrm{g} / 1$ y FIGR $>140 \mu \mathrm{g} / \mathrm{l}$ eritroeitos. Se estimó que la arremía era imputable a deficiencias de hierro si la reducción de Hb se acompañaba de otros dos parámetros indicadores de la nutrición de hierro anormales (Sat, PLE o FS). La anemia se atribuyó a déficit de folato cuando se asoció a concentración de FKGR inferior a $140 \mu \mathrm{g} / \mathrm{l}$ y la etíología combinada se planteó cuando exjstieron evidencias de deficiencia de hierro y de ácido fólico según los criterios onteriormente mencionados. Por el contrario, la anemia que no era encasillable a las etiologías antetiores se la calificó como consecuencia de la desnutrición.

E1 peso y talla de admisión se expresaron como puntuación $Z$, calculados en base a los valoreș de las tablas NCHS.

\section{Resultados}

Treinta y cuatro niños exan del sexo fementno. Su edad: $10,5 \pm 3,8(6-21)$ meses, el peso de nacimiento: $2801 \pm 525(1050.3750) \mathrm{g}$, siendo inferior a $2500 \mathrm{~g}$ en $17 \operatorname{casos}(25,8 \%)$. La puntuación $Z$ del peso de $-3,2 \pm 0,6(-4,9$ a $-2,0)$ y talla de $-2,9 \pm 0,8(-4,7$ a $-1,1)$. El peso para la edad estaba en 33 lactantes (33,3\%) entre -2 y -3 desviaciones estándares, en el resto bajo -3 desviaciones estándares. Los resultados hematológicos se aprecian en la tabla $\mathrm{y}$ figura. Anemia se observó en $53 \%$ de los casos, siendo ésta de carácter leve, con la mayoría de los valores de hemoglobina de los sujetos anémj. cos entre 9 y $10,9 \mathrm{~g} / \mathrm{dl}$. La $\mathrm{Hb}$ se correlacionó directamente con el puntaje $\mathrm{Z}$ de peso de nacimiento $(r=0,34 ; p=0,058)$, puntaje $Z$ de peso para la edad $(r=0,35 ; p=0,0045)$, puntaje $Z$ de talla para la edad $(r=0,26 ; p=0,037)$ y

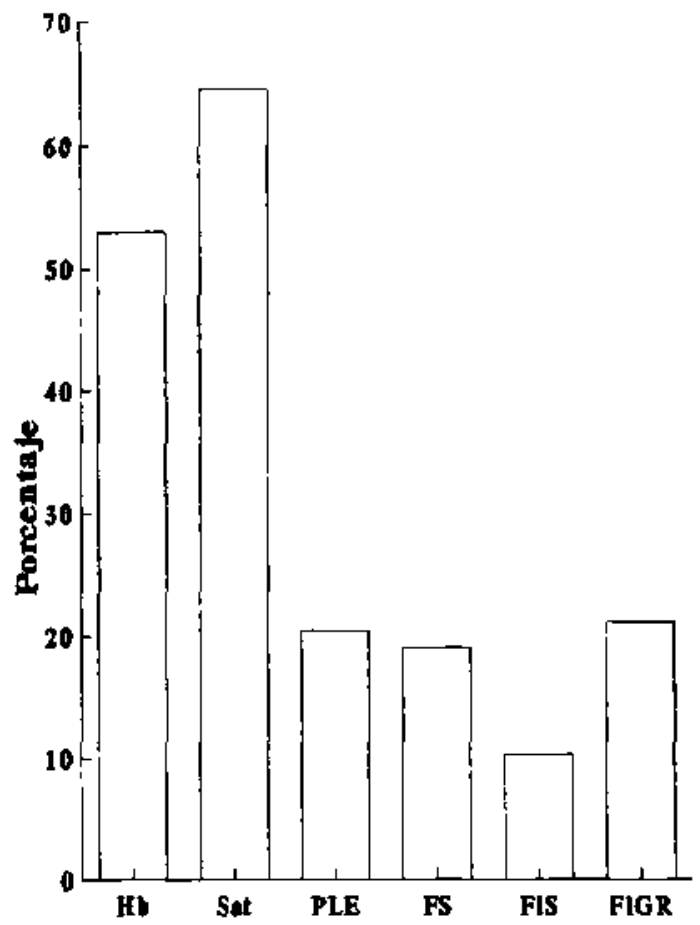

Figura: Prevalencia de valores anormales de hemaglobjna, saturación de la transferrina, protoporfirina libre eritrocitaria, ferritina sérica, folato sérico y folato erjtrocitario.

concentración de hierro sérico $(r=0,24 ; p$ $=0,05)$ e inversamente con la PLE $(r=-0,55$; $p=0,0000$ ). La frecuencia de valores anormales de Sat fue $64,6 \%$, PLE $20,4 \%$, FS $19 \%$, FIS $10,3 \%$ y FlGR $21 \%$.

De las etiologías imputables a la anemia, la desnutrición fue la más frecuente $(46,7 \%)$, seguida de los déficit de hierro $(30 \%)$, ácido fólico $(16,7 \%)$ y carencia combinada de hierro $y$ folato $(6,7 \%)$.

\section{Comentario}

La anemia que se asocia a la desnutrición primaria es un fenómeno complejo debido a que las condiciones que llevan a la desnutrición no sólo determinan restricción del aporte de proteínas, calorías o ambas, sino también de otras vitami. nas y minerales que intervienen en la eritropoye. sis $^{1,3-9}$. Por otra parte las infecciones, que frecuentemente afectan al lactante desnutrido, son capaces de producir reducción de la concentra. 
Tabla

Características hematológicas al ingreso de los 66 lactantes desnutridos

\begin{tabular}{lcr}
\hline & $\begin{array}{c}\text { Promedio } \pm \\
\text { Desviación estandar }\end{array}$ & Rango \\
\hline $\mathrm{Hb}(\mathrm{g} / \mathrm{al})$ & $11,0 \pm 1,2$ & 7,3 a 13,4 \\
$\mathrm{Fe}(\mu \mathrm{g} / \mathrm{d})$ & $35,8 \pm 16,4$ & $\mathbf{1 1 , 1}$ a 79,5 \\
TIBC $(\mu \mathrm{g} / \mathrm{d} 1)$ & $390 \pm 66$ & 220 a 580 \\
Sat $(\%)$ & $9,5 \pm 5,0$ & 2,8 a 28,4 \\
PLE $(\mu \mathrm{g} / \mathrm{dl} \mathrm{GR})$ & $87 \pm 35$ & $\mathbf{4 2}$ a 211 \\
$\mathrm{FS}(\mu \mathrm{g} /)^{*}$ & $21,8(8,9-53,7)$ & $\mathbf{0}$ a 261 \\
FIS $(\mu \mathrm{g} / 1)^{*}$ & $7,9(3,2-19,7)$ & 1 a 51 \\
FIGR $(\mu \mathrm{g} / 1)^{*}$ & $275(135-561)$ & 58 a 1033 \\
\hline
\end{tabular}

* Promedio geométrico y margen de 1 desviación estándar.

ción de la hemoglobina y bloqueo del metabolismo del hierro ${ }^{10}$. La mayoría de los estudios exis. tentes en la literatura han sido realizados en desnutridos de tipo kwashiorkor o marasmo-kwashiorkor, existiendo menor información en desnutridos marásmicos. La combinación de diversos factores participantes en la anemia que acompaña a la desnutrición varía considerablemente según el tipo de desnutrición, edad del sujeto, hábitos alimentarios y explica a su vez las diferencias observadas en la etiología del trastorno en distintos países, a lo que se suma el de no ser posible estudiar aisladamente la participación exclusiva de los déficit calórico o proteico como variables exclusivas, excepto en modelos animales, en que todas las variables puedan ser controladas.

La anemia que acompaña al desnutrido kwashiorkor se debe a menor producción y disminución de la sobrevida de los eritrocitos ${ }^{1,5,11-14}$. La hemólisis estaría determinada por aumento de la permeabilidad ${ }^{1}$ o de la lipoperoxidación de la membrana eritrocitaria o ambas ${ }^{5,13}$. La lipoperoxidación ha sido atribuida a défícit de selenio y vitamina $E^{5}$. Por otra parte, la reduc. ción de la eritropoyesis se debería a alteración de la sintesis de RNA en el eritroblasto ${ }^{15}$ y no a menor producción de eritropoyetina ${ }^{1,11}$. Participan, asimismo, en la génesis de esta anemia otras carencias específicas de vitaminas y minerales (especialmente hierro y ácido fólico, originadas en ingestión insuficiente, malabsorción, aumento de las pérdidas gastrointestinales y reducción de las proteínas transportadoras) ${ }^{1,5 \rightarrow, 11,12}$ como, en parte, las infecciones que frecuentemente afectan a estos sujetos. En animales se ha sugerido que la "anemia", asociada al marasmo, podría corresponder a un fenómeno adaptativo, en que la disminución de la eritropoyesis sería consecuencia de la reducción del número de precursores eritroides, debida al descenso de las demandas de transporte de oxígeno, determinado, a su vez, por las menores necesidades metabólicas de estos sujetos y no a una falta de nutrientes específicos o menor producción de eritropoyetina ${ }^{15-17}$. Por otra parte, los estudios en animales y humanos han demostrado que la sobrevida eritrocitaria es nomal ${ }^{18}$. Según nuestros resultados, la frecuencia de anemia en lactantes desnutridos es alta (53\%), bastante superior a la de $28 \%$ descrita en lactantes eutróficos ${ }^{19}$. En aproximadamente la mitad de los casos no se encuentra carencia específica como causal de la anemia $y$, por tanto, ella podría ser atribuida al aludido efecto adaptativo en desnutridos marásmicos. Entre los factores etiológicos reconocidos que siguen en importancia, aunque con menor frecuencia, están la carencia de hierro y la defjciencia de ácido fólico. En nuestro estudio no analizamos el rol de otros déficit específicos, de más baja prevalencia, que podrían contribuir a la anemia del desnutrido, siendo una de éstas la carencia de cobre ${ }^{20}$, como tampoco el efecto de las infecciones.

En Chile la frecuencia de carencia de hierro en lactantes desnutridos marásmicos, si bien no es despreciable, es bastante más baja que la encontrada en lactantes bien nutridos ${ }^{19,}$ 21, no ocurriendo lo mismo con la carencia de ácido fólico, de bastante mayor prevalencia en lactantes marásmicos ${ }^{9}$ y casi inexistente en eutrófi$\cos ^{22}$.

Las infecciones, incluso leves, son capaces de producir reducción transitoria de las concentraciones de hemoglobina, hierro sérico y satu- 
ración de la transferrina, junto con aumento de la ferritina sérica ${ }^{23}$. Estas modificaciones persisten hasta tres semanas después de iniciado el proceso infeccioso, apareciendo algunas de estas alteraciones desde el período de incubación. A pesar de que los desnutridos no presentaron signos clínicos de infección, por la metodología utilizada no fue posible descartar infecciones subclínicas o las que se hubieran presentado en las semanas previas a la admisión. Sin embargo, la discordancia paradojal en la frecuencia de valores anomales de saturación de transferrina $(64,6 \%)$ y ferritina $(19 \%)$ pudiera, al menos en parte, ser explicada por el efecto de afecciones de este orden.

En la terapia nutricional del desnutrido no sólo se debe considerar un adecuado aporte de proteínas y calorias, sino que también de nutrientes específicos, ya que al reanudarse el crecimiento se intensifican o se hacen manifiestas estas carencias, especialmente las de hierro, ácido fólico y cobre $\mathrm{e}^{1, \mathrm{~B}, \mathrm{z})}$.

De nuestros resultados podemos concluir que la anemia del desnutrido es un fenómeno frecuente, cuya etiología es multifactorial, participando en su génesis la restricción calórica proteica, las infecciones y deficiencias de rutrientes específicos, especialmente hjerro y ácido fólico. Esta es más severa en los lactantes de menor peso de nacimiento $y$ en los con mayor déficit de peso o talla para la edad.

\section{Resumen}

Con la finalidad de describir la prevalencia y etiología de la anemia del desnutrido se seleccionaron al ingreso a un centro de recuperación nutricional 66 lactantes desnutridos marásmicos de 6 a 21 meses de edad, cuyo peso para la edad fuese -2 desviaciones estándares bajo el promedio de las tablas del Centro Nacional de Estadisticas para la Salud de EUA, albúmina sérica $>3,5 \mathrm{~g} / \mathrm{dl}$ y proteinemia $>6 \mathrm{~g} / \mathrm{dl}$. Todos los sujetos tenían una desnutrición primaria debida a ingesta insuficiente de alimentos. Dentro de los 3 dias de admisión se midieron la hernoglobina ( $\mathrm{Hb}$ ), hierro sérico $(\mathrm{Fe})$, capacidad total de combinación de hierro, saturación de la transferrina (Sat), protoporfirina libre eritrocitaria (PLE), ferritina sérica (FS), folato sérico (F1S) y eritrocitario (FlGR). En 53\% de los sujetos se encontró anemia $(\mathrm{Hb}<11 \mathrm{~g} / \mathrm{dl})$, la ma- yoría de carácter leve, $64,6 \%$ presentó Sat $<10 \%, 20,4$ PLE $>120 \mathrm{~g} / \mathrm{dl}$ GR entre los $6 \mathrm{a}$ 11 meses y $>100 \mu \mathrm{g} / \mathrm{dl}$ GR desde los 12 meses, $19 \%$ FS $<10 \mu \mathrm{g} / 1,10,3 \%$ FIS $<3 \mu \mathrm{g} / 1$ y $21,1 \%$ FIGR $<140 \mu \mathrm{g} / \mathrm{l}$ GR. La Hb se correlacionó con las puntuaciones $Z$ de peso/edad $(r=0,35$; $\mathrm{p}<0,005)$ y talla/edad $(\mathrm{r}=0,26 ; \mathrm{p}<0,04)$, $\mathrm{Fe}(\mathrm{r}=0,24 ; \mathrm{p}=0,05)$ y PLE $(r=-0,55$; $\mathrm{p}<0,0001)$. En $46,7 \%$ de los casos la anemia era explicable por desnutrición, $30 \%$ por deficiencia de hierro, 16,7\% por carencia de ácido fólico y $6,7 \%$ por deficiencia combinada de hierro y folato.

(Palabras claves: Desnutrición, deficiencia de hierro, deficiencia de ácido fólico.)

\section{Referencias}

1. MacDougall LG, Moodley G, Eyberg C, Quirk M: Mechanisms of anemia in protein-energy malnutrition in Johannesburg. An I Clin Nuts 1982; $35: 229-235$.

2. Fischer DS, Price DC: A simple serum iron method using the new sensitive chromogen trypiridyl-striazine. Clin Chem 1964; 10:21-30.

3. Heller $S R$, Labbé RF, Nutter JA: A simplified assay for porphyrins in whole blood. Cts Chem 1971:17: 525-528.

4. Waters $A H_{\text {, Mollin }} D L$ : Studies of the folic acid activity of human serum. I Clin Path 1961; 14: $335-344$

5. Fondu $P$, Hariga-Muller $C$, Mozes $N$, Neve J, Van Steirteghem A, Mandelbaum IM: Proteinenergy malnutrition and anemia in Kivu. Am J Clin Nutr $1978 ; 31: 46-56$.

6. Omer A. El Shazali H, Awad El Karim O, El Hossan $A M$ : Studies on the anaemia of kwashiorkor and marasmus in Sudan. J Trop Pediatr Environ Child Health $1973 ; 19: 91-97$.

7. Matoih Y, Zamir R, Bar-Shani S, Grossowicz $N$ : Studies on folic acid in infancy. II. Folic and folinic acid levels in infants with diarrhea, malnntrition, and infection. Pediatrics 1964; 33 : 694-699.

8. Halsted C. Sourial $N$, Guindi S, Abdel Hadi Mourad $K$, Kattab $A K$, Carter JP, Patwardhan VN: Anemia of kwashiorkor in Cairo: deficiencies of protein, iron, and folic acid. Am J Clin Nutr 1969; 22: 1371-1382.

9. Olivares $M$, Hertrampf $E$. Llaguno $S$, Chadud $P$. Stekel A: Folic acid nutrition in marasmic infants. Nutr Res 1986; 6: 1365-1370.

10. Lee GR- The anemia of chronic disease. Semin Hematol 1983; 20:61-80.

11. Fondu $P$, Hofgd $P$, Halvorsen $S$ : The regulation of erythropoiesis in protein-energy-malnutrition. Brit I Haematol 1978; $38: 29-36$.

12. Khalil $M$. Awwad $\boldsymbol{H}$, Hafez $M$ : Plasma and red cell iron turnover in protein calorie malnutrition. Arch Dis Child 1969;44: 124-130. 
13. Fondu $P$, Mozes $N$, Neve $P$, Soher-Robazza $L$, Mondelbaum I: The erythrocyte membrane disturbances in protein-energy malnutrition: nature and mechanjsms. Brit J Haematol 1980; 44 : 605618.

14. Shahidi NT, Diamond LK, Shwachman H: Anemia associated with protein deficiency. J Pediatr 1961; 59: 533-542.

15. Perretta M, Garrido F, Escárate P: Effect of ery thropotetin and testosterone on bone marrow RNA synthesis in rats with protein-energy or protein malnutrition. Nuts Res 1988; 8: 1421. 1429.

16. Stekel A, Smith NJ: Hematologic studies of severe undernutrition of infancy. $\mathbf{l}$. The anemia of prolonged caloric deprivation in the pig. Pediat Res $1969 ; 3: 320-337$.

17. Stekel $A$, Smith NJ: Hematologic studies of severe undernutrition of infancy. II. Erythropoietic response to phlebotomy by calorie-deprived pigs. Pediat Res $1969 ; 3$ : 338-345.

18. Siekel A. Smith NJ: Hematologic studies of severe undernutrition of infancy. 1II. Erythrocyte survival in marasmic infants and calorie deprived pigs. Am J Clin Nutr 1970; 7: 896-904.

19. Rios $E$, Olivares $M, A m a r ~ M$, Chadud P, Pizarro $F$, Stekel A: Evaluation of iron stratus and prevalence of iron deficiency in infants in Chile. In: Nutrition internention strategies in national development. Underwood BA, ed. New York: Academic Press, $1983 ; 273-283$.

20. Cordano A, Baent IM, Groham $C G$ : Copper deficjency in infancy, Pediatrics $1964 ; 34: 324-$ 336.

21. Sandoval G. Winter A, Taboada H, Becker A: Importancia del hierro en la recuperación del desnutrido. Rev Chil Pediatr 1989; 60: 84-88.

22. Olivares $M$. Anderson $M$, Llaguno $S$, Stekel $A$ : Folato sérico y eritrocitario en el lactante. Rev Chil Pedigtr 1993; 54: 246-248.

23. Olivares $M$, Walter $T$, Osorio M, Chadud P, Schlesinger $L$ : Anemia of a mild viral infection: the measles vaccine as a model, Pediatrics 1989; 84: 851-855.

\section{AVISO A LOS AUTORES}

Por acuerdo del Comité Editorial, la Revista Chilena de Pediatría devolverá sin tramitar todos los trabajos que no den estricto cumplimiento al Reglamento de Publicaciones y a las Instrucctones a los Autores que se edtan en cada número de la Revista. 